\title{
Avaliação dos Parâmetros do Modelo de Vazamentos Para Setores de Rede da Cidade de São Carlos - SP
}

\author{
Luisa Fernanda Ribeiro Reis, Adilson dos Santos, Rodrigo de Oliveira Caliman, \\ Fernando das Graças Braga da Silva \\ Departamento de Hidráulica e Saneamento/ EESC-USP - CEP 13566-590 - São Carlos, S.P. - fernanda@sc.usp.br
}

Recebido: 20/11/02 - revisado: 02/05/03 - aceito: 26/06/03

\begin{abstract}
RESUMO
A maior consciência dos aspectos ambientais apresenta reflexos sobre a necessidade de racionalizar o uso dos recursos naturais água e energia. Assim, vários trabalhos vêm sendo desenvolvidos com o objetivo de redução das perdas por vazamento em sistemas de distribuição de água para abastecimento. Entretanto, o sucesso que eles podem alcançar depende de quão realísticos são os parâmetros empregados para expressar tais perdas como função das pressões atuantes no interior dos respectivos sistemas. Da mesma maneira, pode-se dizer que o controle operacional efetivo dos sistemas urbanos de abastecimento de água requer avaliações com o suporte de modelos matemáticos de previsão de comportamento, que produzam repostas dos referidos sistemas em termos das perdas por vazamento, quando expressivas, face às diversas condiçoes de operação a que os mesmos podem ser submetidos. O objetivo deste trabalho é reportar nossa experiência em determinar os valores para os parâmetros do modelo pressão-vazamento, com base nos testes noturnos de vazamentos realizados para setores da rede de abastecimento de água de São Carlos, SP.
\end{abstract}

Palavras chave: vazamentos, abastecimento urbano, redes de distribuição.

\section{INTRODUÇÃO}

O acesso à água constitui questão geradora de inúmeros conflitos e disputas entre os diversos setores econômicos que competem pelo seu uso: o abastecimento doméstico, a indústria e a irrigação, dentre outros. Entretanto, se analisado sob a ótica da sustentabilidade dos recursos hídricos, tão nocivo quanto a falta de água, pode ser o problema causado pelo seu uso inadequado. É, portanto, inaceitável que as perdas por vazamento nos sistemas de distribuição possam atingir porcentagens consideráveis do volume total bombeado e quimicamente tratado para o propósito de abastecimento urbano. Daí a necessidade de estudos visando o controle efetivo de tais perdas. Os vazamentos em sistemas de distribuição de água para abastecimento estão relacionados a fatores como o tipo de solo, qualidade da água, tecnologia e materiais usados na construção da rede, pressão de operação, idade do sistema e práticas de operação e manutenção. Ocorrem, tipicamente, quando os condutos apresentam fraturas circunferenciais ou longitudinais, corrosão, conexões pobres ou comprimidas. As falhas nos registros podem ocorrer como conseqüência de defeitos relacionados ao produto em si, os quais contribuem diretamente com as perdas. $\mathrm{O}$ descuido na instalação pode levar a conexões não estanques, comprometendo todo o sistema hidráulico.

As perdas físicas em redes de distribuição de águas para abastecimento podem ser divididas em duas categorias distintas: visíveis e inerentes, sendo as perdas visíveis aquelas detectáveis em campo, através de instrumentos de detecção de vazamentos, que podem ser facilmente reparadas. Já as perdas inerentes são aquelas que não podem ser detectadas e reparadas com a mesma facilidade, devido a fatores tais como a magnitude e a distribuição das mesmas ao longo das tubulações. Assim, as sistemáticas de controle de vazamentos visam a redução das perdas enquadradas nessa última categoria.

Não é prático reparar todo vazamento em um sistema de distribuição, mesmo que eles possam ser apontados. Tubulações antigas apresentam pequenos vazamentos em todas as juntas e tentativas de repará-los equivaleriam a substituir o conduto principal. Assim, diversos trabalhos (Germanopoulos e Jowitt, 1989; Jowitt e Xu, 1990; Reis et al., 1997; Gueli e Pezzinga, 1998; Vairavamoorthy e Lumbers, 1998) visam a redução de perdas por vazamento em redes de abastecimento de água, através do uso estratégico de VRPs (válvulas redutoras de pressão). Todos eles pressupõem a existência de uma relação conhecida entre vazamento e pressão de serviço na rede em estudo, na forma:

$$
L_{i j}=a \cdot l_{i j} \cdot P_{i j}^{b}
$$

Sendo: $\mathrm{L}=$ vazamento no trecho que conecta os nós i e $\mathrm{j}$ $(\mathrm{L} / \mathrm{s}), l=$ comprimento do conduto ij $(\mathrm{m})$, e $\mathrm{P}=$ carga de pressão média no trecho $\mathrm{ij}(\mathrm{m})$. Os valores para os parâmetros $a$ e $b$ geralmente são tomados de experimentos condu- 
zidos no Reino Unido (Goodwin, 1980) em distritos de operação com pouco ou nenhum consumo noturno, segundo os quais reduções progressivas no vazamento foram registradas para reduções de pressão correspondentes. Tais testes sugerem a relação do tipo apresentado na equação (1), com valor do parâmetro $\mathrm{b}=1,18$. Já o valor de a dependeria das características da rede em estudo. Observa-se que a equação (1) vem sendo empregada indistintamente para expressar o comportamento de setores de rede como um todo e trechos de rede.

Walters e Randall-Smith (2000) realizaram a setorização de redes com propósitos de redução das perdas por vazamento, utilizando a equação ajustada por Lambert (1993) para a mesma curva:

$$
L I=0,5 A Z N P+0,0042 A Z N P^{2}
$$

Onde: $\mathrm{LI}=$ índice de vazamento, $\mathrm{AZNP}=$ pressão noturna média do setor. A estimação da variação na taxa de vazamentos para dada variação na pressão noturna média do setor era baseada na equação (3):

$$
L_{2}=\frac{L I_{2}}{L I_{1}} L_{1}
$$

Sendo: $L_{2}, L_{1}=$ taxas de vazamento posterior e anterior à variação na pressão noturna média do setor, respectivamente; $L I_{2}, L I_{1}=$ índices de vazamento posterior e anterior à variação de pressão na pressão noturna média do setor, respectivamente.

Dessa maneira, o sucesso da redução dos vazamentos preconizado pelas aplicações acima referidas depende de quão realísticas são as relações expressas por (1) e (2).

O presente trabalho tem como objetivo reportar a experiência da nossa equipe de pesquisadores na quantificação de perdas inerentes em dois sub-sistemas urbanos, com base em levantamentos de campo, bem como o seu comportamento face às diferentes pressões de serviço atuantes na rede, com o propósito de gerar subsídios ao controle das mesmas. Os estudos aqui reportados compreenderam as etapas de identificação de sub-áreas isoláveis para estudo, levantamentos, geofonamento, reparo dos vazamentos detectados, testes noturnos de campo sob diferentes níveis de pressão e determinação dos referidos parâmetros $a$ e $b$, condizentes com a realidade dos sistemas estudados.

\section{QUANTIFICAÇÃO DOS VAZAMENTOS}

A quantificação das perdas em sistemas de distribuição pode ser realizada através de medidas em duas escalas: macromedição e micromedição. A macromedição é usada para quantificar os volumes recebidos, transportados e distribuídos. Estas informações são básicas para a elaboração de projetos, construção, operação, manutenção e administração das empresas distribuidoras de água. A micromedição serve para quantificar o consumo do usuário e elaborar o boleto de pagamento, racionalizar o consumo e proporcionar o equilíbrio entre o consumo e a produção.

A literatura reporta tentativas de estabelecer critérios para avaliar perdas através de análise estatística dos dados de consumo doméstico e monitoramento de seções representativas isoladas da rede de distribuição de água. Uma avaliação do sistema de distribuição fornece informações confiáveis, que incluem: avaliação dos hidrômetros, detecção e quantificação dos vazamentos, inventários do sistema, análise de procedimento de contabilidade, medição de produção e pressões, avaliação da eficiência do sistema de bombeamento, e estimativa dos diâmetros internos e dos coeficientes de rugosidade reais. De acordo com Arreguín e Ochoa (1997), esse procedimento pode ser dispendioso para um primeiro estágio de avaliação de perdas porque requer a inspeção de toda rede de distribuição e pode não visar áreas de grandes perdas. Uma amostragem e avaliação simplificadas poderiam reduzir estes custos e representam alternativas viáveis. Assim, propõem um método para avaliar as perdas da rede de distribuição. $\mathrm{O}$ procedimento ajuda a assegurar que os resultados sejam estatisticamente representativos e é potencialmente menos dispendioso do que outras técnicas de avaliação de perdas.

$\mathrm{Na}$ avaliação de perdas nas linhas principais e secundárias, Hueb (1985) combina a teoria simples estratificada com distritos hidrométricos (DHs). Estes índices de perda são determinados à noite, quando geralmente tem-se o mínimo de consumo de água. Cada distrito pode ser isolado, do ponto de vista hidráulico, através do fechamento de válvulas. Uma estação de medida é instalada na seção de válvula usada para abastecer o distrito, conforme ilustra a Figura 1.

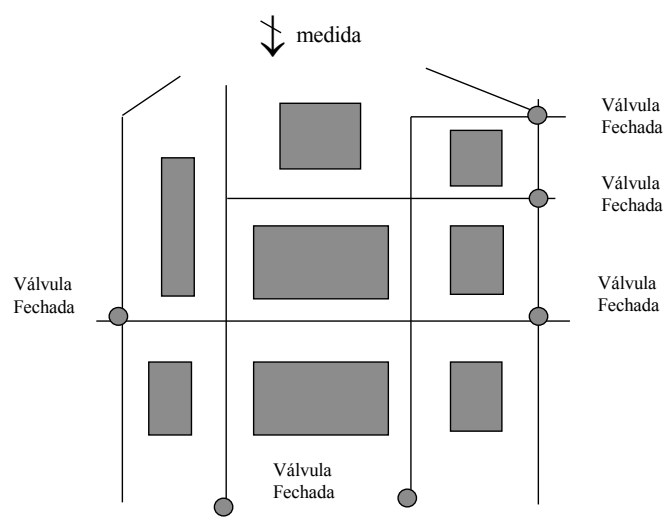

Figura 1 - Distrito Hidrométrico (DH) típico para amostragem. 
Desse modo, DHs são unidades básicas para a amostragem, considerando-se que: a cidade pode ser divida em setores de semelhança sócio-econômica, por existir uma forte relação entre o referido fator e os hábitos de consumo de água. Tais setores podem ser divididos em sub-setores homogêneos com respeito à freqüência anual de vazamentos nas linhas principais e secundárias da rede; e um $\mathrm{DH}$, representativo pode ser selecionado internamente a ele.

A identificação da relação entre pressão de serviço e vazamento, a partir de testes noturnos, implica na necessidade da identificação dos consumos em tais períodos. Sabe-se que a relação entre as perdas físicas e a variação de pressão é função das condições operacionais do sistema em análise. Vários coeficientes e indicadores importados da experiência de outros países vêm sendo utilizados. Entretanto, em função do elevado índice de perdas nos sistemas de distribuição de águas brasileiros, há necessidade de estudos para determinação dos coeficientes e indicadores que reflitam a realidade de tais sistemas.

Khadam et al. (1991) realizaram estudo de vazamentos em 5 (cinco) áreas da cidade de AL-Riyadh, Arábia Saudita. Todos as áreas onde vazamentos foram identificados foram submetidas a pressões de testes para a quantificação dos mesmos. Após o isolamento de cada área pelo fechamento das válvulas apropriadas, a água foi bombeada nas pressões de 2, 4 e 6 bars durante 30, 15 e 15 minutos, respectivamente. Obtiveram-se assim cinco gráficos representativos do vazamento em função da pressão. Observouse que as curvas pressões vazamento não seguiram uma lei exponencial única. Os autores apontam como os fatores responsáveis pela não uniformidade nestas relações para diversos setores:

- Variação de pressão durante o dia, causando mudança nas dimensões das fissuras, particularmente em tubos de polietileno (PE) e Cloro Poli Vinil (PVC), e, conseqüentes variações no tamanho dos orifícios com a pressão;

- Fadiga térmica devido às mudanças extremas de temperatura, provocando defeitos em tubos de PE e PVC e causando fissuras e orifícios de formas diversas; e

Mão de obra pouco qualificada empregada na construção das redes de distribuição.

Sabesp (1998) apresenta resultados de um estudo realizado no Brasil no sentido de quantificar perdas e estabelecer a referida relação. Utilizando-se o distrito pitométrico Vila Deodoro e atendo-se à rede de distribuição, ligações e ramais até a caixa d'água, com 20,5 km de extensão e 4.459 ligações. Segundo Sabesp (1998), a equação para vazão através de um orifício:

$$
\text { Vazão }=\mathrm{A} * \mathrm{C}_{\mathrm{d}} * \mathrm{p}^{\mathrm{N} 1}
$$

representa um modelo adequado para descrever vazamentos diversos numa rede de distribuição. $\mathrm{Na}$ equação (2): $\mathrm{A}=$ área do orifício; $\mathrm{C}_{\mathrm{d}}=$ coeficiente de descarga. $\mathrm{O}$ valor do expoente N1 dependerá da forma dos orifícios. Por exemplo, $\mathrm{N} 1=0.5$, quando a área do vazamento não varia com a pressão como no caso de furos ou trincas em tubos ferro fundido ou ferro galvanizado, furos de PVC ou PAD (polímeros de alta densidade)); N1= 1,15 para redes de distribuição como um todo; N1>1,15 para trincas de PAD e PVC, podendo chegar a 2,5. Os vazamentos com N1 = 2,5 afloraram rapidamente e logo são detectados e reparados. Portanto, para a maioria dos testes, o valor de N1 estaria entre 0,5 e 1,5 . Lembrando ainda que os vazamentos (visíveis, não visíveis ou inerentes) variam em função das pressões e da variação ou não das áreas através das quais ocorrem, a relação geral entre a pressão no ponto representativo da sub-rede e as perdas físicas pode ser representada pela equação (3).

\section{DETERMINAÇÃO DA RELAÇÃO PRESSÃO- VAZAMENTO}

A literatura não fornece indicações claras quanto à metodologia para ajuste da relação pressão-vazamento, a partir de ensaios noturnos. Dessa maneira, neste trabalho foram testadas duas aproximações para o problema. O primeiro método é baseado na variação de pressão obtida em pontos representativos da rede. O segundo faz uso de técnicas de otimização para determinação dos valores $a$ e $b$ através do modelo inverso de minimização dos desvios entre os valores das variáveis de estado monitoradas (pressões e vazões) em campo e os correspondentes valores obtidos através de simulação hidráulica das redes.

Método 1 - Cálculo com base nos pontos representativos por meio de ajuste de curva: Tendo em vista que se dispõe de diversos pares de vazões de abastecimento ao setor e pressões em pontos representativos para o cálculo de dois parâmetros, $a$ e $b$, da equação 1 , pode-se avaliá-los através de ajuste de curva por mínimos quadrados, mantendo-se a suposição com respeito ao comprimento total da sub-rede e consumo desprezível.

Método 2 - Busca Direta: Nesse caso, dois métodos foram empregados, o método dos Algoritmos Genéticos (Michalewicz, 1989) e a busca univariada (Fox, 1971) dos valores $a$ e $b$, por razões de simplicidade, para realizar a minimização dos quadrados dos desvios entre os valores de pressão e vazão monitorados em campo e simulados através de modelo hidráulico, em pontos estratégicos da rede e na entrada do setor, respectivamente. Detalhes a cerca dos métodos, bem como dos operadores e parâmetros utilizados pelos AGs, podem ser obtidos nos referidos trabalhos. 
A função objetivo utilizada na otimização foi expressa por:

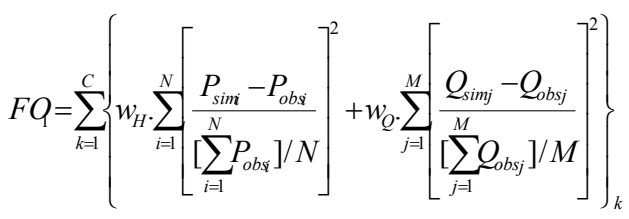

Sendo:

$P$ - carga de pressão (m);

$Q$ - vazão $(\mathrm{L} / \mathrm{s})$

$N$ - conjunto de pontos de observação de valores de pressão

$M$ - conjunto de pontos de observação de valores de vazão

C - número de condições permanentes obtidas para as diferentes modulações da válvula da entrada do setor.

$\mathrm{w}_{\mathrm{H}}, \mathrm{w}_{\mathrm{Q}}$ - pesos atribuídos aos desvios de carga e vazão

sim, obs - denotam valores simulados e observados, respectivamente.

O problema de otimização pode então ser expresso como: $\min _{a, b} F O_{1}$ (no caso da busca univariada) ou $\max _{a, b} F O_{1}{ }^{-1}$ (no caso dos AGs).

O modelo de simulação hidráulica utilizado tem como base o sistema de equações de balanço de massa nodal expresso por:

$$
\sum_{j \in R_{i}} Q_{i j}+0.5 \sum_{j \in R_{i}} Q S_{i j}+C_{i}=0 ; \quad i=1,2, \ldots N
$$

sendo: $L_{i j}$ representa o vazamento no conduto que liga os nós $i$ e $j, Q_{i j}$ representa a vazão $(\mathrm{L} / \mathrm{s}), C_{i}$ o consumo do nó $i, H_{i}$ a carga hidráulica no nó $i, N$ é o número de nós com carga hidráulica desconhecida e $R_{j}$ representa o conjunto de nós conectados a i.

No modelo, a Fórmula Universal foi incorporada para representar a relação entre perda de carga e vazão, por representar uma formulação mais geral, válida para diferentes tipos de escoamento.

$$
\Delta H=0,0827 \frac{f Q^{2} L}{D^{5}}
$$

onde: $\Delta H$ representa perda de carga $(\mathrm{m}) ; Q$ representa a vazão $\left(\mathrm{m}^{3} / \mathrm{s}\right) ; D$ diâmetro do conduto $(\mathrm{m}) ;$ l comprimento do conduto (m); $f$ fator de atrito.

O fator de atrito $f$, foi expresso através da expressão proposta por SWAMEE (1993), válida para os escoamentos, laminar, turbulento liso, de transição e turbulento rugoso:

$$
f=\left\{\left(\frac{64}{\operatorname{Re} y}\right)^{8}+9,5\left[\ln \left(\frac{\varepsilon}{3,7 D}+\frac{5,74}{\operatorname{Re} y^{0,9}}\right)-\left(\frac{2500}{\operatorname{Re} y}\right)^{6}\right]^{-16}\right\}^{0,125}
$$

A relação não linear entre vazamento e pressão de serviço foi incorporada pela equação (1), aqui reproduzida:

$$
L_{i j}=a l_{i j}\left(P_{i j}\right)^{b}
$$

onde : $L_{i j}$ representa o vazamento através do conduto $\ddot{j} ;$ a e $b$ são os parâmetros relação vazamento $\mathrm{x}$ pressão, $l_{i j} \mathrm{o}$ comprimento do conduto $i j$ e $P_{i j}$ a pressão média no interior do conduto $i j$, calculada como:

$$
P_{i j}=0.5\left[\left(H_{i}-G_{i}\right)+\left(H_{j}-G_{j}\right)\right]
$$

onde : $G_{i}$ representa a cota topográfica do nó $i$.

Observa-se que a unidade de a depende da magnitude do expoente $b$ (para $b=1, a=L / s . m)$. Assim sendo, omite-se a unidade de a no presente texto.

\section{ESTUDOS REALIZADOS}

Os estudos aqui reportados compreenderam as etapas de identificação de sub-áreas isoláveis de interesse, levantamentos, geofonamento, reparo dos vazamentos detectados e testes noturnos de campo sob diferentes níveis de pressão, além das atividades de simulação computacional para levantamento da curva pressão-vazamento, em conformidade com os métodos já descritos.

\section{Identificação das sub-áreas isoláveis para estudos}

Investigações iniciais visaram identificar setores de rede passíveis de serem isolados, os quais apresentassem também vazamentos expressivos. Assim, foram eleitos os setores de rede denominados Jardim Monte Carlo e São Carlos III, esboçados nas Figuras 2 e 3, integrantes do sistema de distribuição de águas para abastecimento da cidade de São Carlos, SP, ambos de características tipicamente residenciais. O Jardim Monte Carlo abastece uma população de aproximadamente 5.072 habitantes, ao longo de seus 9.173 metros de extensão, com tubulações de ferro fundido e P.V.C., dotadas do total de 1.051 ligações/economias (Junho de 1999). A rede de distribuição do Setor São Carlos III possui $23.060 \mathrm{~m}$ de extensão, em PVC e cimento amianto e é dotada de 2.052 ligações (Dezembro de 2000), que abastecem uma população aproximada de 10.000 habitantes.

No caso do Jardim Monte Carlo, uma adutora que reforçava o abastecimento do setor precisou ser isolada, fechando-se o seu registro, de maneira a manter um único ponto de alimentação de vazão para o setor. No São 
Carlos III, houve a necessidade de interrupção do fornecimento de água para o Bairro de Santa Felícia, durante os testes, para eliminar a necessidade de medidas da vazão normalmente derivada para esse setor. Além disso, construiu-se uma caixa de visita para monitoramento de vazão em um ponto intermediário da rede do São Carlos III.

\section{Levantamentos}

Além dos dados normalmente coletados (consumos mensais por quadra e dados das redes propriamente ditas), foram realizados estudos das vazões noturnas e da incidência histórica de vazamentos e da localização dos reparos efetuados, com base nos boletins de reparos das ocorrências efetuados em resposta às reclamações dos consumidores.

\section{Geofonamento e reparo dos vazamentos detectados}

A equipe foi treinada para detectar vazamentos, com auxílio de equipamentos como haste de escuta e geofone eletrônico, identificando pontos suspeitos para posterior confirmação e reparo pela equipe do Serviço Autônomo de Águas e Esgoto (SAAE) de São Carlos, antes da realização dos testes noturnos.

\section{Testes de vazamentos noturnos}

Os testes noturnos de vazamentos foram realizados com o auxílio de equipamentos como medidor de vazão ultrassônico e data loggers de pressão. Com base na topografia do terreno e no traçado da rede, foram selecionados pontos para aquisição de dados simultâneos, nas proximidades de nós de naturezas diversas, indicados nas Tabelas 1 e 2 . Como o alvo do trabalho era a determinação das perdas por vazamento, os ensaios de campo aqui reportados foram realizados durante o período noturno, mais especificamente da 01:00 às 04:00 horas, durante o qual a vazão registrada se deve a um consumo desprezível e vazamentos expressivos, dada à ocorrência de pressões mais elevadas no interior dos condutos. Tais experimentos consistiram em variar a pressão nos setores através de manobras de fechamento gradual da VRP (válvula redutora de pressão) e do registro de gaveta, existentes nas linhas de alimentação do Jardim Monte Carlo e do São Carlos III, respectivamente, produzindo os registros simultâneos das Fig.4,5 e 6.

\section{RESULTADOS E DISCUSÃO}

Constatou-se que, embora tivéssemos informações de que ambos os setores analisados apresentassem vazamentos expressivos, o JMC apresenta vazão noturna superior ao SC III.

Os levantamentos mostraram que as áreas de maior incidência de vazamentos correspondem às de maiores pressões. As análises realizadas com o propósito de localização dos vazamentos, baseadas no relato das peças

substituídas, apontaram também os percentuais de 67\% e $33 \%$ das ocorrências de vazamentos em ramais prediais e na rede, respectivamente. Tais valores reforçam a hipótese de que os vazamentos inerentes podem ser considerados distribuídos ao longo das linhas adutoras e, da mesma forma que os consumos, concentrados nos nós da rede para efeito da simulação hidráulica.

Os dados registrados durante os testes noturnos do JMC (Jardim Monte Carlo) e do SC III (São Carlos III) são ilustrados nas figuras 4 e 5 e 6 , respectivamente, onde observam-se quatro condições quase permanentes de vazão e pressão, através dos patamares bem delineados, correspondendo às condições de abertura total dos respectivos dispositivos de controle de alimentação aos setores, e manobras de fechamento aproximado gradativo de $1 / 4,1 / 2 \mathrm{e}$ $3 / 4$.

Tabela 1 - Pontos de monitoramento - JMC.

\begin{tabular}{|c|c|c|c|}
\hline NÓ & $\begin{array}{c}\text { NATURE- } \\
\text { ZA }\end{array}$ & $\begin{array}{c}\text { LOCALIZAÇÃO } \\
\text { - COTA (m) }\end{array}$ & MEDIDA \\
\hline $56 a$ & $\begin{array}{l}\text { Entrada do } \\
\text { setor }\end{array}$ & $\begin{array}{c}\text { Antes da VRP } \\
\text { (PV)- 855,0 }\end{array}$ & Pressão \\
\hline $56 \mathrm{~b}$ & $\begin{array}{l}\text { Entrada do } \\
\text { setor }\end{array}$ & $\begin{array}{c}\text { após a VRP (PV) } \\
-855,0\end{array}$ & $\begin{array}{c}\text { Vazão de distri- } \\
\text { buição e pres- } \\
\text { são }\end{array}$ \\
\hline 57 & Médio 1 & CR $-834,0$ & Pressão \\
\hline 7 & Crítico & CR $-853,7$ & Pressão \\
\hline 19 & Crítico & CR $-857,3$ & Pressão \\
\hline 26 & Médio 2 & CR $-837,6$ & Pressão \\
\hline 38 & Médio 3 & $\mathrm{CR}-836,5$ & Pressão \\
\hline 51 & Extremo & CR - 816,4 & Pressão \\
\hline
\end{tabular}

Tabela 2 - Pontos de monitoramento- SC III.

\begin{tabular}{cccc}
\hline N & NATU- & LOCALIZAÇÃO- & MEDIDA \\
Ó & REZA & COTA $(\mathrm{m})$ & \\
\hline 65 & Crítico & $\mathrm{CR}-868,8$ & Pressão \\
12 & Médio 2 & $\mathrm{CR}-862,4$ & Pressão \\
20 & Extremo & $\mathrm{CR}-853,7$ & Pressão \\
76 & Médio 1 & $\mathrm{CR}-865,5$ & Pressão \\
48 & Médio 3 & $\mathrm{CR}-858,3$ & Pressão \\
16 & Extremo & $\mathrm{CR}-846,7$ & Pressão \\
87 & Crítico & $\mathrm{CR}-872,7$ & Pressão \\
14 & Crítico & JR $-873,0$ & Pressão e Vazão \\
\hline JR = Tubulação ponto de jusante do reservatório de \\
alimentação; CR =Cavalete residencial
\end{tabular}




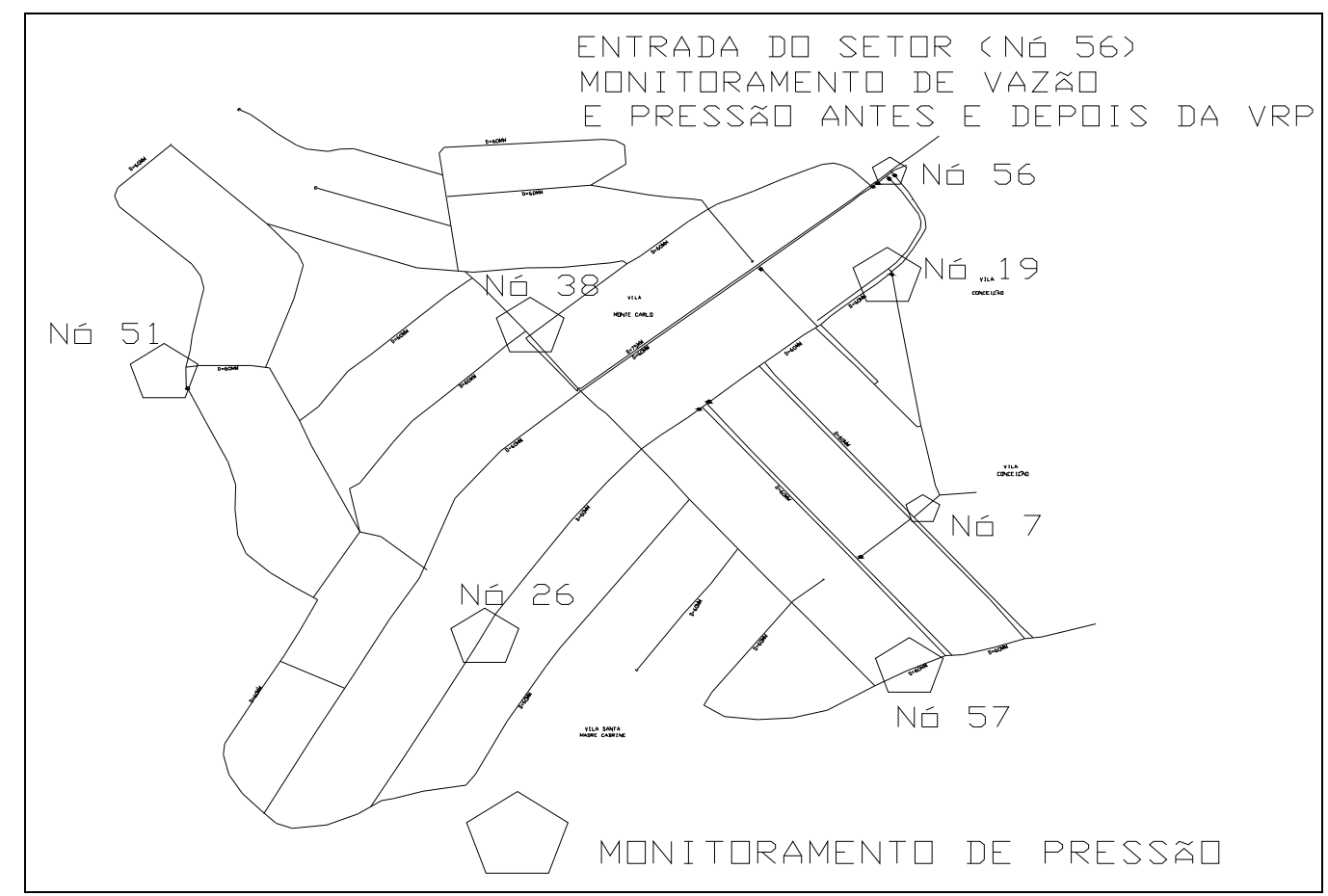

Figura 2 - Esboço do Setor Jardim Monte Carlo.

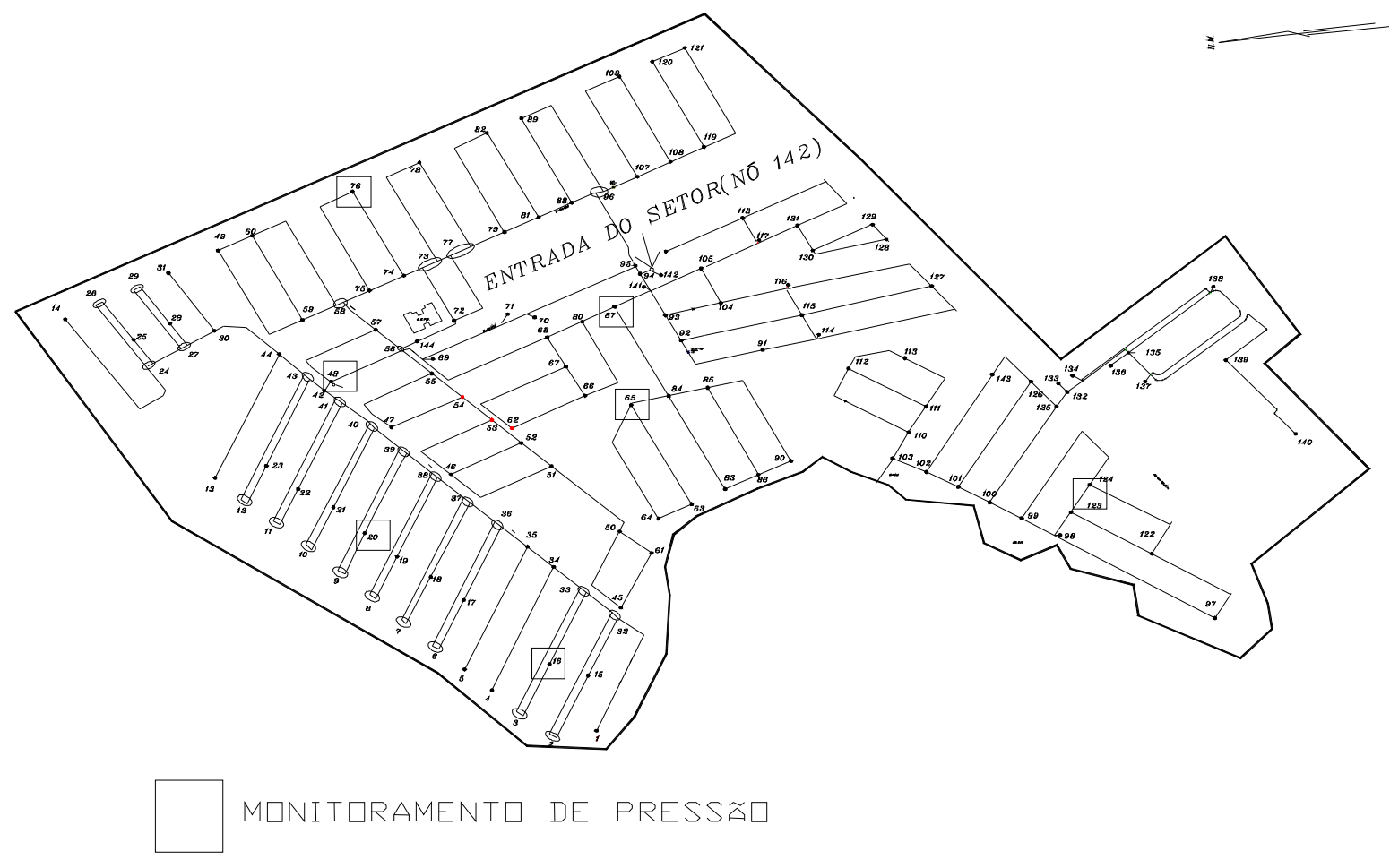

MUNITGRAMENT DE VAZ $\square$ NA ENTRADA D SETUR

Figura 3 - Esboço do Setor São Carlos III. 


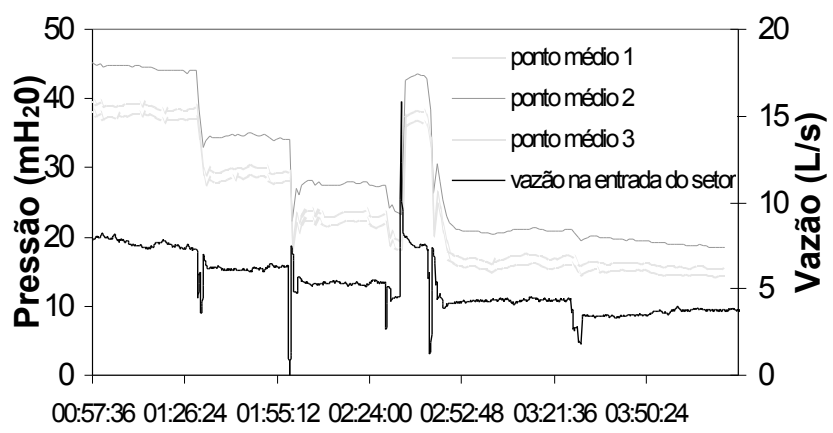

Hora do dia 24-06-1999

Figura 4 - Registros de vazão e pressões - ensaio noturno do JMC.

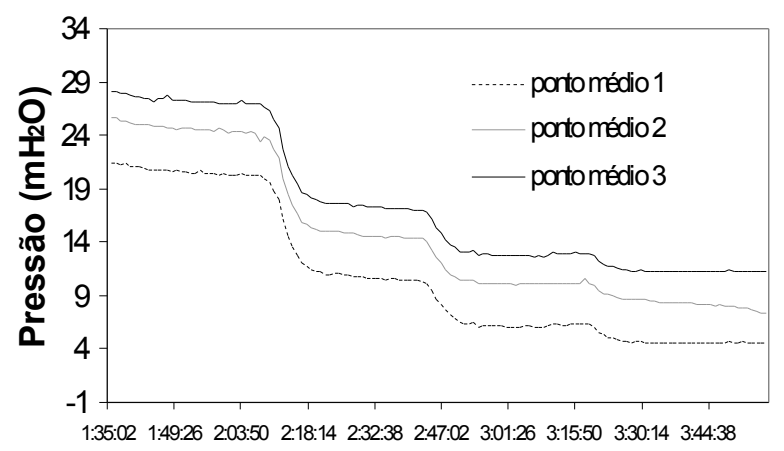

Hora do dia 06-12-00

Figura 5 - Registros de pressões-ensaio noturno do SC III.

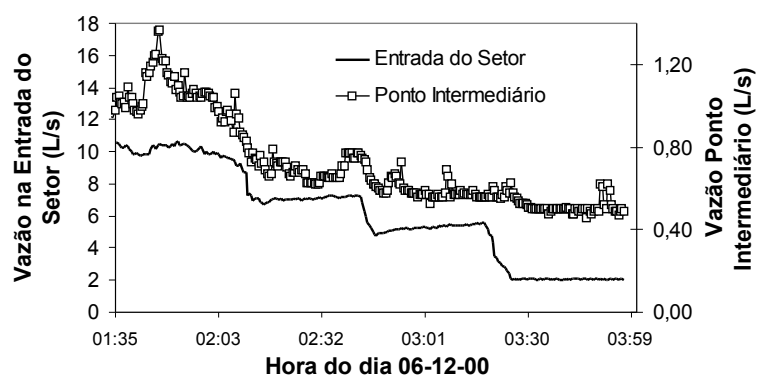

Figura 6 - Registros de vazão - ensaio noturno do SC III.

Os valores de vazão e pressão relativos aos patamares constam das Tabelas 3 e 4, possibilitando as estimativas dos parâmetros $a$ e $b$, segundo as duas aproximações descritas.

\section{Avaliações segundo o primeiro método}

Os cálculos segundo o primeiro método foram efetuados considerando nós de naturezas distintas como sendo representativos para ambos os setores, conforme os valores ajustados das Tabelas 3 e 4, que representam as curvas das Figuras 7 e 8 . Dados relativos a pressões negativas regis- tradas foram descartados durante as análises, tendo em vista a imprecisão de medidas na presença de ar.

Para o JMC, os valores de $b$ ficaram na faixa compreendida entre 0,267 e 1,21, enquanto os valores de $a$ foram estimados entre $3,48.10^{-4}$ e $6,18.10^{-6}$. Já no caso do SC III, os valores de $b$ ficaram na faixa compreendida entre 0,333 e 1,440 , enquanto os valores de $a$ foram estimados entre $1,24.10^{-4}$ e $7,40.10^{-6}$.

Tabela 3 - Ajuste do modelo para pontos de naturezas distintas da rede JMC.

\begin{tabular}{ccccc}
\hline NÓ & NATUREZA & \multicolumn{3}{c}{ AJUSTE } \\
\cline { 3 - 5 } & & $\mathrm{a}$ & $\mathrm{b}$ & $\mathrm{R}^{2}$ \\
57 & Médio 1 & $4,86.10^{-5}$ & 0,746 & 0,997 \\
7 & Crítico & $3,38 \cdot 10^{-4}$ & 0,277 & 0,971 \\
19 & Crítico & $3,48 \cdot 10^{-4}$ & 0,267 & 0,970 \\
26 & Médio 2 & $6,72.10^{-5}$ & 0,683 & 0,995 \\
38 & Médio 3 & $7,75 \cdot 10^{-5}$ & 0,651 & 0,995 \\
51 & Extremo & $6,18.10^{-6}$ & 1,210 & 0,999 \\
\hline
\end{tabular}

Tabela 4- Ajuste do modelo para pontos de naturezas distintas da rede SC III.

\begin{tabular}{ccccc}
\hline NÓ & NATUREZA & \multicolumn{3}{c}{ AJUSTE } \\
\cline { 3 - 5 } & & $\mathrm{a}$ & $\mathrm{b}$ & $\mathrm{R}^{2}$ \\
65 & Crítico & $1,24.10^{-4}$ & 0,417 & 0,996 \\
124 & Médio 2 & $3,72.10^{-5}$ & 0,755 & 0,999 \\
20 & Extremo & $7,40.10^{-6}$ & 1,140 & 0,995 \\
76 & Médio 1 & $7,59.10^{-5}$ & 0,564 & 1,000 \\
48 & Médio 3 & $2,39.10^{-5}$ & 0,861 & 0,999 \\
16 & Extremo & $1,99.10^{-6}$ & 1,440 & 0.989 \\
87 & Crítico & $1,68.10^{-4}$ & 0,333 & 0,993 \\
\hline
\end{tabular}

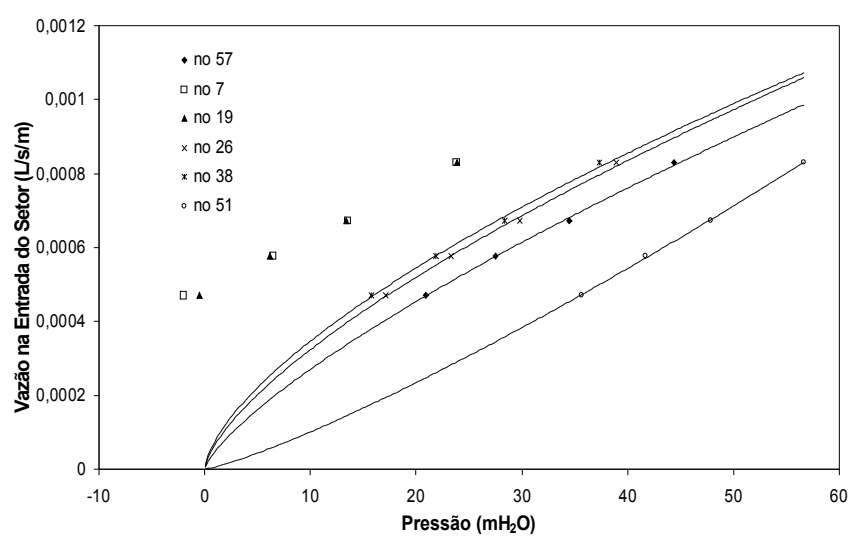

Figura 7- Ajuste para pontos de naturezas distintas - JMC. 


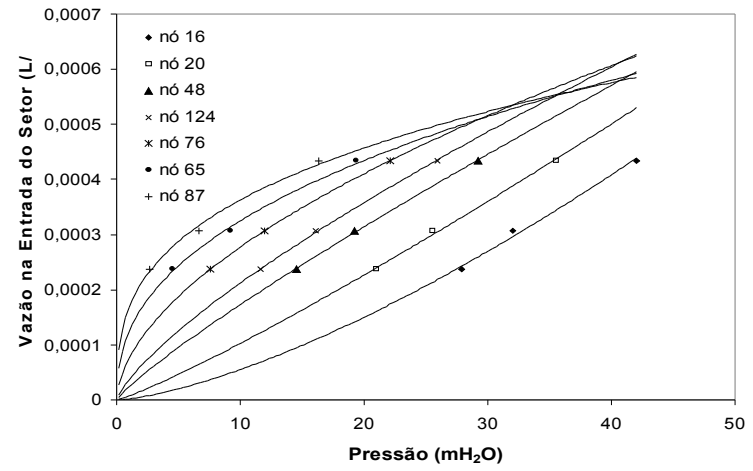

Figura 8- Ajuste para pontos de naturezas distintas - SC III.

O exame das curvas ajustadas sugere que, para ambos os setores, funções com expoentes $b$ entre 1,15 (Miyaoka e Funabashi, 1984) e 1,18 representam curvas intermediárias dentre as delineadas, para as quais valores $a$ de mesma ordem de grandeza dos reportados na literatura $\left(10^{-5}\right)$ seriam obtidos. Assim, a maior dificuldade inerente a esse método diz respeito à identificação dos pontos representativos da pressão noturna dos setores, devido ao desconhecimento das cargas de pressão da rede a priori.

\section{Avaliações de acordo com o segundo método}

Reconhece-se que os valores $a$ e $b$ buscados devam resultar de um procedimento de minimização dos desvios entre os valores observados e obtidos através de simulação hidráulica para variáveis de estado chaves como pressões e vazões. Entretanto, tais resultados podem estar sujeitos a distorções consideráveis se os parâmetros imputados para simulação das redes, tais como rugosidades, estiverem distantes da realidade de campo. Assim, o modelo inverso expresso por (6) deve ser considerado como uma das etapas do procedimento computacional de calibração de redes cujos vazamentos representam parcela considerável da vazão de alimentação. Os resultados obtidos através do modelo de otimização descrito devem, portanto, ser considerados com cautela.

Parâmetros Globais - JMC: Os métodos de busca univariada (Santos, 2000) e AGs (Caliman, 2002) foram utilizados para a realização das estimativas dos parâmetros a e b globais para o JMC, cujos resultados são resumidos nas Tabelas 5 e 6 .

Parâmetros específicos para as áreas de características diferentes - JMC: Com o objetivo de produzir parâmetros mais realísticos, foi realizada a divisão do setor em duas zonas, levando-se em consideração as cotas topográficas do setor, mapa de incidência de vazamentos e o material das tubulações (PVC ou ferro fundido), nos moldes sugeridos por Tucciarelli et al. (1999). A zona 1 caracteriza- se por apresentar PVC na maioria dos condutos e cotas topográficas mais elevadas e a zona 2 por apresentar ferro fundido na maioria dos condutos e baixas cotas topográficas.

As Tabelas 7 e 8 possibilitam a análise comparativa entre os valores simulados e observados de vazão e pressão, admitindo-se duas zonas distintas. Tais resultados foram obtidos via AGs.

Tabela 5 - Resultados obtidos para $a$ e $b$ globais - JMC

\begin{tabular}{|c|c|c|c|c|c|c|c|}
\hline Busca & F.O. & $\begin{array}{c}a \\
10^{-5}\end{array}$ & b & & $\begin{array}{c}\text { Vazão } \\
\text { Obs. } \\
(\mathrm{I} . / \mathrm{s})\end{array}$ & $\begin{array}{c}\text { Vazão } \\
\text { Calc. } \\
\text { (T./s) }\end{array}$ & $\begin{array}{r}\text { Desvio } \\
(\%)\end{array}$ \\
\hline \multirow{3}{*}{ Univ. } & \multirow{3}{*}{0,2347} & \multirow{3}{*}{6,97} & \multirow{3}{*}{0,67} & 1 & 7,61 & 7,96 & 4,59 \\
\hline & & & & 2 & 6,16 & 6,59 & 6,98 \\
\hline & & & & 3 & 5,28 & 5,53 & 0,95 \\
\hline \multirow{3}{*}{ AGs } & \multirow{3}{*}{0,2398} & \multirow{3}{*}{9,79} & \multirow{3}{*}{0,54} & 1 & 7,61 & 7,55 & 0,79 \\
\hline & & & & 2 & 6,16 & 6,43 & 4,38 \\
\hline & & & & 3 & 5,28 & 5,52 & 4,55 \\
\hline
\end{tabular}

Tabela 6 - Valores de pressão observados e calculados parâmetros $a$ e $b$ globais JMC

\begin{tabular}{|c|c|c|c|c|c|}
\hline \multirow[b]{2}{*}{ Nó } & \multirow[b]{2}{*}{$\begin{array}{c}\text { Pressão } \\
\text { Obs. } \\
\text { (m) }\end{array}$} & \multicolumn{2}{|c|}{ AGs } & \multicolumn{2}{|c|}{ Busca Univariada } \\
\hline & & $\begin{array}{c}\text { Pressão } \\
\text { Calculada } \\
\text { (m) }\end{array}$ & $\begin{array}{c}\text { Des- } \\
\text { vio } \\
(\%)\end{array}$ & $\begin{array}{c}\text { Pressão } \\
\text { Calculada } \\
\text { (m) }\end{array}$ & $\begin{array}{c}\text { Desvio } \\
(\%)\end{array}$ \\
\hline \multicolumn{6}{|c|}{ Manobra 1} \\
\hline 57 & 44,39 & 45,34 & 2,15 & 45,16 & 1,73 \\
\hline 7 & 23,79 & 25,76 & 8,28 & 25,65 & 7,81 \\
\hline 19 & 23,82 & 23,61 & 0,88 & 23,6 & 0,92 \\
\hline 26 & 38,88 & 42,21 & 8,56 & 41,96 & 7,91 \\
\hline 38 & 37,35 & 41,25 & 10,44 & 41,04 & 9,44 \\
\hline 51 & 56,65 & 61,84 & 9,16 & 61,55 & 8,64 \\
\hline \multicolumn{6}{|c|}{ Manobra 2} \\
\hline 57 & 34,44 & 35,27 & 2,71 & 35,30 & 2,20 \\
\hline 7 & 13,58 & 15,57 & 14,65 & 15,53 & 14,35 \\
\hline 19 & 13,38 & 13,19 & 1,42 & 13,19 & 1,42 \\
\hline 26 & 29,80 & 32,23 & 8,15 & 32,12 & 4,42 \\
\hline 38 & 28,37 & 31,18 & 9,90 & 31,09 & 9,58 \\
\hline 51 & 47,85 & 51,88 & 8,42 & 51,75 & 8,50 \\
\hline \multicolumn{6}{|c|}{ Manobra 3} \\
\hline 57 & 27,48 & 28,24 & 2,77 & 28,23 & 2,72 \\
\hline 7 & 6,46 & 8,46 & 30,96 & 8,46 & 30,95 \\
\hline 19 & 6,18 & 5,92 & 4,20 & 5,92 & 4,02 \\
\hline 26 & 23,26 & 25,25 & 8,56 & 25,22 & 8,42 \\
\hline 38 & 21,90 & 24,15 & 10,27 & 24,12 & 10,13 \\
\hline 51 & 41,66 & 44,93 & 7,85 & 44,87 & 7,70 \\
\hline
\end{tabular}


Tabela 7 - Resultados obtidos para $a$ e $b$ diferenciados JMC dividido em duas zonas

\begin{tabular}{ccc}
\hline & $\mathrm{a}$ & $\mathrm{b}$ \\
& $10^{-5}$ & \\
\hline Zona 1 & 7,54 & 1,11 \\
\hline Zona 2 & 0,99 & 0,55 \\
\hline
\end{tabular}

Tabela 8 - Pressões observadas e simuladas- JMC dividido em duas zonas

\begin{tabular}{|c|c|c|c|}
\hline Nó & $\begin{array}{c}\text { Pressão } \\
\text { Obs. } \\
(\mathrm{m})\end{array}$ & $\begin{array}{c}\text { Pressão } \\
\text { Calculada } \\
(\mathrm{m})\end{array}$ & $\begin{array}{c}\text { Desvio } \\
(\%)\end{array}$ \\
\hline \multicolumn{4}{|c|}{ Manobra 1 } \\
\hline 57 & 44,39 & 45,61 & 2,75 \\
\hline 7 & 23,79 & 26,05 & 9,50 \\
\hline 19 & 23,82 & 23,67 & 0,63 \\
\hline 26 & 38,88 & 41,85 & 7,64 \\
\hline 38 & 37,35 & 40,03 & 7,18 \\
\hline 51 & 56,65 & 58,37 & 3,04 \\
\hline \multicolumn{5}{|c|}{ Manobra 2 } \\
\hline 57 & 34,44 & 35,53 & 3,16 \\
\hline 7 & 13,58 & 15,80 & 16,36 \\
\hline 19 & 13,38 & 13,24 & 1,05 \\
\hline 26 & 29,80 & 32,09 & 7,68 \\
\hline 38 & 28,37 & 30,43 & 7,26 \\
\hline 51 & 47,85 & 49,62 & 3,70 \\
\hline \multicolumn{5}{|c|}{ Manobra 3 } \\
\hline 57 & 27,48 & 28,46 & 3,57 \\
\hline 7 & 6,46 & 8,63 & 33,59 \\
\hline 19 & 6,18 & 5,95 & 3,72 \\
\hline 26 & 23,26 & 25,21 & 8,38 \\
\hline 38 & 21,90 & 23,65 & 8,00 \\
\hline 51 & 41,66 & 43,34 & 4,03 \\
\hline
\end{tabular}

Parâmetros Globais - SCIII: O métodos de AGs (Caliman, 2002) foi utilizado para a realização das estimativas dos parâmetros a e b globais para o SCIII, cujos resultados são resumidos nas Tabelas 9 e 10.

Tabela 9 -Parâmetros Globais - SC III

\begin{tabular}{cccc}
\hline & Vazão & Vazão Calculada & Desvio (\%) \\
\hline 1 & 7,61 & 7,87 & 3,41 \\
2 & 6,16 & 6,54 & 6,17 \\
3 & 5,28 & 5,59 & 5,87 \\
\hline F.O & 0,1605 \\
\hline
\end{tabular}

TABELA 10- Resultados obtidos para a e b globais para o setor SC III

\begin{tabular}{ccccccc}
\hline F.O. & $\begin{array}{c}\text { a } \\
\left(10^{-5}\right)\end{array}$ & b & Manobra & $\begin{array}{c}\text { Vazão } \\
\text { Obs. } \\
(\mathrm{L} / \mathrm{s})\end{array}$ & $\begin{array}{c}\text { Vazão } \\
\text { Calc. } \\
(\mathrm{L} / \mathrm{s})\end{array}$ & $\begin{array}{c}\text { Des- } \\
\text { vio } \\
(\%)\end{array}$ \\
\hline \multirow{3}{*}{0,0507} & \multirow{3}{*}{4,04} & \multirow{2}{*}{0,74} & 1 & 10,09 & 10,11 & 0,20 \\
\cline { 3 - 7 } & & & 7,09 & 7,03 & 0,85 \\
\hline
\end{tabular}

TABELA 11 - Resultados obtidos para parâmetros globais para todo o setor SC III

\begin{tabular}{|c|c|c|c|}
\hline Nó & $\begin{array}{c}\text { Pressão } \\
\text { Obs. } \\
(\mathrm{m})\end{array}$ & $\begin{array}{c}\text { Pressão } \\
\text { Calculada } \\
(\mathrm{m})\end{array}$ & $\begin{array}{c}\text { Desvio } \\
(\%)\end{array}$ \\
\hline \multicolumn{3}{|c|}{ Manobra 1} \\
\hline $\mathbf{1 6}$ & 42,04 & 41,49 & 1,31 \\
\hline $\mathbf{2 0}$ & 35,55 & 34,61 & 2,64 \\
\hline $\mathbf{4 8}$ & 29,23 & 30,07 & 2,87 \\
\hline $\mathbf{1 2 4}$ & 25,93 & 26,08 & 0,58 \\
\hline $\mathbf{7 6}$ & 22,08 & 22,89 & 3,67 \\
\hline $\mathbf{6 5}$ & 19,39 & 19,59 & 1,03 \\
\hline $\mathbf{8 7}$ & 16,32 & 15,87 & 2,76 \\
\hline \multicolumn{3}{|c|}{ Manobra 2} \\
\hline $\mathbf{1 6}$ & 32,04 & 31,47 & 1,78 \\
\hline $\mathbf{2 0}$ & 25,55 & 24,55 & 3,91 \\
\hline $\mathbf{4 8}$ & 19,23 & 20,02 & 4,11 \\
\hline $\mathbf{1 2 4}$ & 16,12 & 15,97 & 0,93 \\
\hline $\mathbf{7 6}$ & 11,93 & 12,83 & 7,55 \\
\hline $\mathbf{6 5}$ & 9,23 & 9,54 & 3,36 \\
\hline $\mathbf{8 7}$ & 6,66 & 5,72 & 14,11 \\
\hline \multicolumn{3}{|c|}{ Manobra 3 } \\
\hline $\mathbf{1 6}$ & 27,87 & 27,13 & 2,66 \\
\hline $\mathbf{2 0}$ & 21,05 & 20,20 & 4,04 \\
\hline $\mathbf{4 8}$ & 14,51 & 15,66 & 7,93 \\
\hline $\mathbf{1 2 4}$ & 11,67 & 11,59 & 0,69 \\
\hline $\mathbf{7 6}$ & 7,55 & 8,47 & 12,19 \\
\hline $\mathbf{6 5}$ & 4,54 & 5,18 & 14,10 \\
\hline $\mathbf{8 7}$ & 2,66 & 1,32 & 50,38 \\
\hline
\end{tabular}

Parâmetros específicos para as áreas de características diferentes - SCIII: Procedimento similar ao JMC foi adotado para produzir parâmetros globais relativos ao SCIII, subdividindo a área em duas e três zonas, sendo que essa última subdivisão apresentou resultados superiores.

As Tabelas 11 e 12 possibilitam a análise comparativa entre os valores simulados e observados de vazão e pressão, admitindo-se três zonas distintas.

Diferentemente dos resultados obtidos para o JMC, os ajustes via modelo inverso produziram desvios 
aceitáveis para os valores de pressão e vazão, de acordo com os padrões internacionais, que estabelecem os limites de $1 \mathrm{~m}$ e 5\%, respectivamente. A única exceção é a pressão observada no nó 87, para a terceira manobra da válvula, que representou uma pressão $1,34 \mathrm{~m}$ acima do valor simulado correspondente.

Tabela 12 - $a$ e $b$ diferenciados SC III-3 zonas

\begin{tabular}{|c|c|c|c|c|c|c|}
\hline & $\begin{array}{c}a \\
.10^{-5}\end{array}$ & $b$ & Manobra & $\begin{array}{c}\text { Vazão } \\
\text { Obs. } \\
(L / s)\end{array}$ & $\begin{array}{c}\text { Vazão } \\
\text { Calculada } \\
(L / s)\end{array}$ & $\begin{array}{c}\text { Desvio } \\
(\%)\end{array}$ \\
\hline Z 1 & 1,25 & 0,49 & 1 & 10,09 & 10,35 & 2,58 \\
\hline Z 2 & 5,78 & 0,60 & 2 & 7,09 & 7,06 & 0,42 \\
\hline Z3 & 7,54 & 0,80 & 3 & 5,46 & 5,39 & 1,28 \\
\hline F.O & \multicolumn{2}{|c|}{0,0400} & & & & \\
\hline
\end{tabular}

Tabela 13-Resultados obtidos para $a$ e $b$ diferenciados SC III-3 zonas

\begin{tabular}{|c|c|c|c|}
\hline Nó & $\begin{array}{c}\text { Pressão } \\
\text { Obs. } \\
(\mathrm{m})\end{array}$ & $\begin{array}{c}\text { Pressão } \\
\text { Calculada } \\
(\mathrm{m})\end{array}$ & $\begin{array}{c}\text { Desvio } \\
(\%)\end{array}$ \\
\hline \multicolumn{4}{|c|}{ Manobra 1} \\
\hline $\mathbf{1 6}$ & 42,04 & 41,87 & 0,41 \\
\hline $\mathbf{2 0}$ & 35,55 & 34,87 & 1,91 \\
\hline $\mathbf{4 8}$ & 29,23 & 29,61 & 1,30 \\
\hline $\mathbf{1 2 4}$ & 25,93 & 25,71 & 0,85 \\
\hline $\mathbf{7 6}$ & 22,08 & 22,51 & 1,95 \\
\hline $\mathbf{6 5}$ & 19,39 & 19,59 & 1,03 \\
\hline $\boldsymbol{8 7}$ & 16,32 & 15,86 & 2,82 \\
\hline \multicolumn{4}{|c|}{ Manobra 2} \\
\hline $\mathbf{1 6}$ & 32,04 & 31,71 & 1,03 \\
\hline $\mathbf{2 0}$ & 25,55 & 24,71 & 3,29 \\
\hline $\mathbf{4 8}$ & 19,23 & 19,79 & 2,91 \\
\hline $\mathbf{1 2 4}$ & 16,12 & 15,80 & 1,99 \\
\hline $\mathbf{7 6}$ & 11,93 & 12,64 & 5,95 \\
\hline $\mathbf{6 5}$ & 9,23 & 9,53 & 3,25 \\
\hline $\boldsymbol{8 7}$ & 6,66 & 5,71 & 14,26 \\
\hline \multicolumn{5}{|c|}{ Manobra 3 } \\
\hline $\mathbf{1 6}$ & 27,87 & 27,32 & 1,97 \\
\hline $\mathbf{2 0}$ & 21,05 & 20,32 & 3,47 \\
\hline $\mathbf{4 8}$ & 14,51 & 15,62 & 7,65 \\
\hline $\mathbf{1 2 4}$ & 11,67 & 11,49 & 1,54 \\
\hline $\mathbf{7 6}$ & 7,55 & 8,34 & 10,46 \\
\hline $\mathbf{6 5}$ & 4,54 & 5,17 & 13,88 \\
\hline $\boldsymbol{8 7}$ & 2,66 & 1,32 & 50,40 \\
\hline \multicolumn{5}{|c|}{} \\
\hline
\end{tabular}

Avaliou-se ainda que, no caso do SCIII, as pressões observadas nos nós de naturezas diversas praticamente correspondem às pressões estáticas dos mesmos, para as três condições operacionais de manobra do registro. Tal fato explica a concordância entre os valores simulados e observados, uma vez que, nessas condições de escoamento, o efeito das perdas de carga, mesmo que para rugosidades irreais, é minimizado.

\section{CONCLUSÕES}

O presente trabalho descreve nossa experiência em determinar os parâmetros do modelo de vazamentos para dois setores da rede de abastecimento da cidade de São Carlos, SP, com base em testes noturnos de vazamentos.

Foram empregados dois métodos para determinação dos valores $a$ e $b$ do modelo: cálculo com base nos pontos representativos por meio de ajuste de curva e método inverso (busca direta).

O primeiro método produziu como resultado dos ajustes, para ambos os setores, valores para o parâmetro $b$ na faixa de 1.15 a 1,18 , representando curvas intermediárias dentre as delineadas, para as quais valores $a$ de mesma ordem de grandeza dos reportados na literatura $\left(10^{-5}\right)$ foram obtidos. Assim, a maior dificuldade desse método mostrou-se ser relativa à identificação dos pontos representativos da pressão noturna dos setores, para a avaliação dos vazamentos totais dos mesmos.

O segundo método utilizado mostrou que, no caso de perdas de carga desprezíveis (valores das pressões observadas próximos das pressões estáticas correspondentes), as determinações realizadas utilizando os dados dos testes noturnos, desconsiderando-se os parâmetros da rede, tais como rugosidades, é razoável. O mesmo não ocorrendo com a rede JMC, para a qual valores das pressões observadas mostraram-se discrepantes das pressões estáticas correspondentes. Assim, os resultados obtidos através do segundo método para os setores de características distintas estudados possibilitaram a conclusão de que os parâmetros do modelo de vazamentos devem ser estimados via procedimento de calibração que vise também a determinação de parâmetros da rede propriamente dita (rugosidades, diâmetros, comprimentos, etc.). A reconhecida importância de se proceder ao ajuste dos parâmetros via simulação, mesmo porque o objetivo é utilizá-los posteriormente em modelos desse tipo, reforça tal recomendação.

Diversas foram as dificuldades encontradas na realização dos estudos de vazamentos, dentre as quais podese apontar a incerteza quanto à eliminação dos vazamentos mais grosseiros, na fase anterior aos ensaios noturnos. Mesmo que todos os reparos necessários tenham sido realizados, não se pode garantir que novos vazamentos não 
tenham surgido no período que antecedeu os testes noturnos, dado o comportamento dinâmico e oculto dos mesmos. Nota-se ainda que novos vazamentos podem surgir como resposta do sistema aos reparos introduzidos.

Outra dificuldade, relacionada mais diretamente ao segundo método adotado, diz respeito à incertezas quanto aos níveis topográficos assumidos, cujas distorções podem representar desvios consideráveis na avaliação dos vazamentos.

\section{AGRADECIMENTOS}

Os autores agradecem à FINEP como agência financiadora do projeto RECOPE - REHIDRO, em que se insere o presente estudo; ao SAAE-São Carlos e sua equipe pelo apoio logístico no desenvolvimento deste trabalho; ao CNPq pela concessão de bolsas de mestrado e de produtividade em pesquisa a autores deste trabalho; à FAPESP, pela bolsa de doutorado destinada ao terceiro autor; e à Sabesp, pela colaboração, possibilitando que tivéssemos acesso ao trabalho desenvolvido pela Companhia.

\section{REFERÊNCIAS}

ARREGUÍN-CORTES, F. I. e OCHOA-ALEJO, L. H. (1997). Evaluation of Water Losses in Distribution Networks. Journal of Water Resources Planning and Management. v.123, n.5, p. 284-291.

CALIMAN, R. (2002) Estudo das Perdas por Vazamento Aplicado a Setores da Rede de Abastecimento de Água de São Carlos, SP, dissertação a ser apresentada à Escola de Engenharia de São Carlos.

FOX, R. L., Optimization Methods for Engineering Design, Addison-Wesley Inc., California, 1971.

GERMANOPOULOS, G. e JOWITT, P.W. (1989). Leakage reduction by excess pressure minimization in a water supply network. Proc. Instn. Civ. Engng, part1, n.87, p.215-224, June.

GOODWIN, S.J. (1980). The results of the experimental programme on leakage and leakage control. WRc Technical Report 154.

GUELI, R.;PEZZINGA, G. (1998). Algoritmi genetici per la regolazione di valvole ai fini della riduzione delle perdite. Atti, XXVI CONV. DI IDRAULICA E CONSTRUZIONI IDRAULICHE, Catania, Italy.

HUEB, A. (1985). Control de fuga en los sistemas de distribuição de agua potable. Lima, Organizacion Pan-americana de la Salud, (CEPIS Manual Dtiapa, 07).

JOWITT, P.W.; XU, C. (1990). Optimal Valve Control in Water Distribution Networks. Journal of Water Resources Planning and Management, v.116, n.4, p.455-472.

KHADAM, M.O.; SHAMMAS, N.KH.; AL-FERAIHEEDI, Y.(1991). Water losses from municipal utilities and their impacts, J. Int. Water Resour. Assn., v.16, n.4, p.254-261.

LAMBERT, A.O. (1993). Controlling leaks with BABE. W\&WT, November, 1993.

MICHALEWICZ, Z. (1994). Genetic Algorithm + data structures $=$ evolution programs, 2a. ed., Springer-Verlag, New York.

MIYAOKA, SH. E FUNABASHI, M. (1984). Optimal Control of Water Distribution Systems by Network Flow Theory, IEEE Transactions on Automatic Control, Vol. AC-29, n. 4, pg. 303-311.

REIS, L.F.R.; PORTO, R.M.; CHAUDRY, F.H. (1997). Optimal location of control valves in pipe networks by genetic algorithm. Journal of Water Resources Planning and Management, v.123, n.6, p.317-326, Nov/Dec.

SABESP(1998). Relatório interno de consultoria técnica para determinação da relação entre perdas físicas e variação da pressão na rede, através de intervenções monitoradas em área piloto da RMSP.

SANTOS, A. (2000). Estudo das Perdas por Vazamento Aplicado a um Setor da Rede de Abastecimento de Água de São Carlos, SP, dissertação apresentada à Escola de Engenharia de São Carlos, 114 p.

TUCCIARELLI, T.; CRIMINISI, A.; TERMINI, D. (1999). Leak Analysis in Pipeline Systems by Means of Optimal Valve Regulation, ASCE, v.125, n.3, p.277-285.

VAIRAVAMOORTHY, K.; LUMBERS, J.(1998). Leakage Reduction in Water Distribution Systems: Optimal Valve Control, Journal of Hidraulic Engineering, v.124, n.11, p.1146-1153.

WALTERS, G. A. e RANDALL-SMITH, M. (2000). In: INTERNATIONAL SYMPOSIUM CWS 2000: Procceedings. Water network Modelling for Optimal Design and Management, Centre for Water Systems, University of Exeter, UK, p. 161-169.

\section{Leakage Studies for Water Supply Network Sec- tors in the City of of São Carlos SP}

\section{ABSTRACT}

Greater environmental consciousness indicates the need to make more effective use of the natural resources water and energy. Thus, several studies have been developed to reduce leakage losses in water supply distribution systems. However, the success that may be achieved depends on the degree of realism of the parameters employed to express leakage as a function of pressure. It could also be said that effective operational control of urban water supply systems requires evaluations supported by mathematical models to forecast behavior, providing answers to these systems in terms of leakage, when they are relevant, in order to deal with the different operational conditions to which they may be submitted. The purpose of this paper is report our experience in determining the values for the pressure-leakage model 
Avaliação dos Parâmetros do Modelo de Vazamentos Para Setores de Rede da Cidade de São Carlos-SP

parameters, based on nocturnal tests conducted in sectors of the water supply network in the city of São Carlos, S.P.

Key-words: Leakage; water supply. 